and assays the effect of livestock vaccination rate on disease report in humans.

Methods Necessary Information was achieved from concerned authorities. Data analysis was done by SPSS software version 16 . Mapping was performed using ArcGIS version 9.3.

Results Brucellosis is reported more in the West and Northwest provinces, where their sheep and goat population density is higher than other parts of the country. The highest incidence of brucellosis was seen in 1991 with a rate 101.63 per one hundred thousand people. On average, each year about 27500 new cases were reported in the country. Brucellosis incidence over the years showed a decreasing trend $(r=-0.79, \mathrm{P}<0.001)$. Pearson correlation analysis showed that in different provinces there was a significant association between the annual incidence of Malta fever and the annual infection rate of sheep and goats $(r=0.347, p<0.001)$, the annual incidence of Malta fever and the annual infection rate of cattle $(\mathrm{r}=0.327, \mathrm{p}<0.001)$ and the annual incidence of Malta fever and the percentage of vaccinated cows $(r=0.098, p=0.031)$.

Conclusion As the prevalence of brucellosis depends very closely with the prevalence of brucellosis in animals, the control of the disease in human population is not possible unless it is controlled in livestock; so the cooperation between different organizations (Veterinary, public health ...) is needed.

\section{P1-256 WHAT NEXT MEASLES CONTROL FOR KARNATAKA INDIA?}

doi:10.1136/jech.2011.142976e.48

R Mugali. ${ }^{*}$ German TB leprosy relief association, Chennai, India

Introduction Worldwide, measles still kills 400 people every day, more than $90 \%$ of them being under-fives. Three out of four deaths happen in India, The objectives of my work are to describe the epidemiology of measles in Karnataka and to identify ways to improve measles control in the state.

Methodology I collated and tabulated weekly surveillance reports and outbreak investigation line lists for 4 years. I synthesised the spatio-temporal distribution of measles cases and outbreaks in the state. Through logistic regression multivariate analysis, specified the contextual demographic and socio-cultural determinants of measles outbreaks and vaccination coverage in the state of Karnataka.

Findings Measles surveillance data from 2006 to 2009 reveal that measles is indeed endemic in Karnataka, with frequent outbreaks. The notification rate of measles is 10.94 cases per 100000 population per year. Seasonality of notified measles cases characteristically increases between November and April and decreases from May to October. There were 163 confirmed outbreaks in the state in the 4 years under study. Measles outbreaks were happening consistently in the northern part of the state and sporadically in the southern part: The duration of these outbreaks from the date of appearance of rash in a first case to last case was ranged from 7 days to 120 days with median of 39 days.

Discussion Significant reduction in measles incidence and outbreaks can be attained only by adding a second dose to the routine vaccination scheme in the whole state and by conducting catch-up campaigns in northern Karnataka.

\section{P1-257 RELATIONSHIP BETWEEN THE NUMBER OF ESTABLISHED CARDIOVASCULAR RISK FACTORS AND ANNUAL MEDICAL COSTS IN JAPAN: COST ANALYSIS IN 38890 JAPANESE INDIVIDUALS}

doi:10.1136/jech.2011.142976e.49

${ }^{1} Y$ Murakami, ${ }^{2} \mathrm{~T}$ Okamura, ${ }^{3} \mathrm{~K}$ Miura, ${ }^{4} \mathrm{H}$ Ueshima. ${ }^{1}$ Department of Medical Statistics, Shiga University of Medical Science, Otsu, Shiga, Japan; ${ }^{2}$ Department of Preventive Medicine and Public Health, Keio University, Shinjuku, Tokyo, Japan; ${ }^{3}$ Department of
Health Science, Shiga University of Medical Science, Otsu, Shiga, Japan; ${ }^{4}$ LifestyleRelated Disease Prevention Center, Shiga University of Medical Science, Otsu, Shiga, Japan

Background Information about the relationship between the number of established cardiovascular disease risk factors and medical costs is limited.

Methods A linked analysis of the medical costs and health examination measures was performed in Shiga, Japan. Medical costs information for the period April 2000-May 2006 was collected from the Shiga National Health Insurance Organization database. Corresponding data for 38890 individuals (age, $\geq 40$ years) generated during annual health examination conducted between 2000 and 2003 were collected from the local municipalities in the Shiga prefecture. These data were merged by name, sex, and dates of birth. Median and interquartile were used to compare the annual medical costs among groups with different number of risk factors. The risk factors were hypertension ( $\mathrm{SBP} \geq 140$ or $\mathrm{DBP} \geq 90$ ), high cholesterol (total cholesterol $\geq 240$ ) and blood glucose levels (casual blood glucose $\geq 126$ ), and smoking.

Results The median total annual medical cost in the group with no risk factors was 217149 Yen for men and 147880 Yen for women. The median total annual medical cost in the group with four risk factors was 1.28 times higher in men (278 651 Yen) and 1.80 times higher in women (265.788 Yen), as compared to that in the group with no risk factors. These trends were most apparent in outpatients (men: 1.47 times (127205 Yen vs 186811 Yen); women: 2.23 times (94648 Yen vs 211123 Yen)).

Conclusion Annual medical costs increase with an increase in the number of cardiovascular disease risk factors in the Japanese population.

\section{P1-258 IS AWARENESS OF DOTS AMONG MEDICAL PRACTITIONERS A WORRY? A DEVELOPING NATION SCENARIO}

doi:10.1136/jech.2011.142976e.50

V Nagaraja, ${ }^{*}$ M A Khan, G B Sankapithilu. Mysore Medical College \& Research Institute, Mysore, Karnataka, India

Background Annually two million people in India develop Tuberculosis and 330000 die. WHO-recommended DOTS strategy was pilot-tested in 1993 and launched as Revised National Tuberculosis Control Programme (RNTCP) in 1997. Awareness of DOTS among the doctors in the private sector was appalling although nationwide coverage was attained by 2006.

Objective To study awareness of DOTS among Medical Practitioners of urban and rural Mysore.

Methodology 401 Medical practitioners in hospitals and nursing homes of urban and rural areas of Mysore who treated Tuberculosis patients (private and public sector) were approached. They were grouped under different specialties as per the year of graduation (before or after introduction of DOTS).

Results Thirty eight per cent doctors who graduated before the introduction of DOTS didn't follow DOTS compared to $14.9 \%$ doctors who graduated later. 100\% doctors working in Government sector felt that DOTS was better than daily regimen while $85 \%$ from the private sector felt so. Only $47.9 \%$ of the doctors in the private sector practiced DOTS compared to $95.1 \%$ in the Govt. Sector. Hence, the number of doctors practicing DOTS in Private Sector was $<50 \%$ of that in the Govt. Sector. Both of these comparisons were found to be statistically highly significant $(p<0.001)$. Awareness of DOTS was alarmingly low among Orthopaedic Surgeons, Gynaecologists and Paediatricians when compared to Physicians and General Practitioners. 
Conclusions DOTS awareness is still low among doctors who graduated before the introduction of DOTS. Private practitioners harboured myths and misconceptions about DOTS.

\section{P1-259 RELATIONS BETWEEN BMI AND TOTAL AND CAUSE SPECIFIC MORTALITY IN JAPAN: AGES COHORT}

\section{doi:10.1136/jech.2011.142976e.51}

${ }^{1} \mathrm{M}$ Nakade, ${ }^{*} \mathrm{~T}$ Ojima, ${ }^{3} \mathrm{H}$ Hirai, ${ }^{4} \mathrm{~J}$ Aida, ${ }^{5} \mathrm{~T}$ Hanibuchi, ${ }^{3} \mathrm{~K}$ Kondo. ${ }^{1}$ Tokaigakuen University, Nagoya, Japan; ${ }^{2}$ Hamamatsu University School of Hamamatsu, Japan; ${ }^{3}$ Nihon Fukushi University, Nagoya, Japan; ${ }^{4}$ University College London, London, UK; ${ }^{5}$ Ritsumeikan University, Kyoto, Japan

Purpose Within OECD countries, Japan has the lowest percentage $(3.4 \%)$ of the people with obesity ( $30 \leqq$ body mass index, BMI). This study aims to reveal the relationship between BMI and mortality among older Japanese.

Method We started a cohort study (Aichi Gerontological Evaluation Study, AGES) on non- institutionalised elderly aged 65 and over in 2003. We excluded the subjects with missing data and who died within 1 year in 4 year follow-up period considering reverse causation. Consequently, 17017 subjects among 21047 respondents for baseline survey were analysed. Cox's proportional hazard model was applied to calculate HR and 95\% CI of BMI for all cause and cause specific mortality. Age, disease history, weight loss, smoking, alcohol drinking, and exercise were used as covariates.

Result Respondents with lowest category of BMI (under 18.5) had the highest all cause mortality among both sexes. Compared to the respondents with BMI 23.0-24.9, men respondents with under 18.5 BMI and BMI 18.5-22.9 had significantly higher BMI (2.16 $(1.58-2.95, \mathrm{p}<0.001)$ and $1.30(1.04-1.63, \mathrm{p}=0.015)$ respectively). For cause specific mortality, men with BMI under 18.5 had a significantly higher $\mathrm{HR}$ of malignant neoplasm mortality $(\mathrm{HR}=1.68$ $(1.05-2.67, \mathrm{p}=0.030))$ and respiratory disease mortality $(\mathrm{HR}=5.63$ $(2.57-12.34, \mathrm{p}<0.001))$.

Conclusion Older Japanese with lower BMI had a significantly higher risk of all cause mortality in both sexes. Not only obesity, but also underweight prevention is needed for older Japanese.

\section{P1-260 DENTAL FLUOROSIS IN CHILDREN OF SÃO PAULO, BRAZIL, IN 1998-2008}

doi:10.1136/jech.2011.142976e.52

${ }^{1} \mathrm{P}$ C Narvai, ${ }^{3} \mathrm{M} C$ Soares, ${ }^{2} \mathrm{~A} C$ Frias, ${ }^{3} \mathrm{R}$ A A Marques, ${ }^{*}{ }^{3} \mathrm{D} S \mathrm{~S}$ Cruz, ${ }^{1} \mathrm{P}$ Frazao, 'J L F Antunes. 'School of Public Health, University of Sao Paulo, Sao Paulo, Brazil; ${ }^{2}$ School of Dentistry, University of Sao Paulo, Sao Paulo, Brazil; ${ }^{3}$ Health Department, Sao Paulo, Brazil

Introduction Water fluoridation and fluoride dentifrice contribute effectively in caries prevention. Both resources have nearly universal coverage in Sao Paulo, Brazil, since the 1980s. However, multiple exposures to fluoride demand the surveillance of dental fluorosis. This study aimed to assess fluorosis prevalence from 1998 to 2008. Methods Epidemiological surveys of oral health conducted in 1998 $(n=244), 2002(n=253)$ and in $2008(n=4249)$ informed fluorosis prevalence among 12-year-old school children. Although using different sample sizes, all surveys were considered representative for the city. Dental examinations were performed at schools, under natural illumination. The assessment of dental fluorosis used the index proposed by Dean in 1942, and recommended by the WHO for oral health surveys in 1997. Data analysis used the comparison of proportions included in the Epi-Info software.

Results Fluorosis prevalence was $43.9 \%$ (95\% CI $37.7 \%$ to $50.1 \%$ ) in $1998,33.2 \%$ (27.7\% to $39.2 \%$ ) in 2002 , and $39.1 \%$ (37.6\% to $40.6 \%$ ) in 2008. Mild and very mild fluorosis accounted for $88 \%$ of cases observed in 1998, $95 \%$ in 2002 , and $94 \%$ in 2008 . Less than $1 \%$ of examined children had severe fluorosis in 1998 and 2008; no cases were observed in 2002. No statistically significant difference was observed for the overall prevalence and for rates that specifically assessed differential levels of fluorosis during the period.

Conclusion The prevalence of dental fluorosis among children in Sao Paulo was stationary in the period 1998 to 2008. Most of the prevalence refers to mild and very mild levels of fluorosis, with no impact in function and aesthetics.

\section{P1-261 PREDICTION OF DROPOUT TUBERCULOSIS TREATMENT ON PRIORITARY CITIES TO CONTROL IN ESPÍRITO SANTO STATE, BRAZIL}

doi:10.1136/jech.2011.142976e.53

T N do Prado, ${ }^{*}$ R Dietze, A R Netto, E Zandonade, E L N Maciel. Universidade Federal do Espírito Santo, Vitoria, Espírito Santo, Brazil

Objectives To identify the epidemiological factors related to the abandonment of TB treatment in the priority municipalities for TB control and to establish a score for use in TB control programs to identify patients most likely to abandon treatment.

Methods case-control study matched by sex and notification place to compare patients who dropped out of the treatment (cases) with those who were cured (control) in eight priority municipalities for TB control in the Espirito Santo state, from January 2006 to July 2008. Patients were interviewed directly by one of the researchers, at the clinic or at home. To data analysis we performed a bivariate analysis and the significant results obtained from these analysis were for the logistic regression analysis and network neural artificial (NNA). The questionnaire score was created and validated.

Results The study involved 21 cases and 41 controls. In the bivariate analysis, the epidemiological factors involved in the TB treatment dropout were identified as follows: average income, smoking, age, occupation, religion, drugs, previous treatment for $\mathrm{TB}$ and willingness to abandon. The logistic regression analysis and neural network revealed that the use of illicit drugs and patient without religion was strongly related to the abandonment. It was found that the neural classification was not more effective than logistic regression for the score marks preparation.

Conclusions The score created was able to estimate the treatment dropout cases identified in the study and can be used in programs of $\mathrm{TB}$ control to identify patients most likely to abandon $\mathrm{TB}$ treatment.

\section{P1-262 POST-MORTEM INFECTION CONTROL IN JAPAN}

doi:10.1136/jech.2011.142976e.54

M Nishiyama,* H Sakuda, H Kawasaki, Y Shigematsu, R Doi, P D’Angelo, M Kakehashi. Hiroshima Bunkyo Women's University, Hiroshima, Japan

Introduction Healthcare workers in Japan lack the required level of understanding when dealing with cadavers at home and have the continuous and conflicting problem of balancing traditional, cultural beliefs with providing high quality infection control methods.

Objective The objectives of this research were to clarify the reasons for poor infection control post-mortem and to statistically show the significance of the problem.

Methods In 2006, 4773 participants gave feedback from a questionnaire. Participants were drawn from 13 different types of healthcare. They completed questionnaires about prevention and control of infection from cadavers. Questions covered four main areas: (1) The management of cadavers, (2) The role of nursing staff, 